\title{
Oral Lactobacillus strains reduce cytotoxicity and cytokine release from peripheral blood mononuclear cells exposed to Aggregatibacter actinomycetemcomitans subtypes in vitro
}

\author{
Nuntiya Pahumunto ${ }^{1}$, Amina Basic ${ }^{2}$, Anna-Karin Östberg ${ }^{2}$, Rawee Teanpaisan ${ }^{1}$ and Gunnar Dahlen²*
}

\begin{abstract}
Background: This study evaluated the effect of oral lactobacilli on the cytotoxicity and cytokine release from peripheral blood mononuclear cells (PBMCs) when exposed to Aggregatibacter actinomycetemcomitans subtypes in vitro. The supernatants and cell wall extracts (CWEs) of eight A. actinomycetemcomitans strains, representing different subtypes, and three Lactobacillus strains were used. The PBMCs from six blood donors were exposed to supernatants and CWES of A. actinomycetemcomitans or Lactobacillus strains alone or combinations and untreated cells as control. The cytotoxicity was determined by trypan blue exclusion method and IL-1 $\beta$ secretion by ELISA. TNF-a, IL-6, and IL-8 secretions were measured using Bioplex Multiplex Immunoassay.

Results: Supernatants or CWEs from all bacterial strains showed cytotoxicity and IL-1 $\beta$ secretion and the subtypes of A. actinomycetemcomitans showed generally a significantly higher effect on PBMCs than that of the Lactobacillus strains. Two highly toxic A. actinomycetemcomitans strains (JP2 and JP2-like) induced a higher response than all other strains. When combined, Lactobacillus significantly reduced the toxicity and the IL-1 $\beta$ secretion induced by $A$. acinomycetemcomitans. The effect varied between the subtypes and the reduction was highest for the JP2 and JP2like strains. The Lactobacillus paracasei strain SD1 had a higher reducing effect than the other Lactobacillus strains. This strain had a consistent reducing effect on all subtypes of $A$. actinomycetemcomitans cytotoxicity, and release of $I L-1 \beta, I L-6, I L-8$, and TNF-a from PBMCs of the blood donors. A strong and significant variation in cytokine release between the six blood donors was noticed.
\end{abstract}

Conclusions: Lactobacillus spp. and L. paracasei SD1 in particular, showed a limited but statistically significant reducing interaction with $A$. actinomycetemcomitans toxicity and release of cytokines in vitro.

Keywords: Microbial interaction, Oral lactobacilli, Aggregatibacter actinomycetemcomitans, Bacterial toxicity, Cytokine release, Peripheral blood mononuclear cells

\footnotetext{
*Correspondence: gunnar.dahlen@odontologi.gu.se

2Department of Oral Microbiology and Immunology, Institute of Odontology, Sahlgrenska Academy, University of Gothenburg, Gothenburg, Sweden

Full list of author information is available at the end of the article
}

(c) The Author(s). 2020 Open Access This article is licensed under a Creative Commons Attribution 4.0 International License, which permits use, sharing, adaptation, distribution and reproduction in any medium or format, as long as you give appropriate credit to the original author(s) and the source, provide a link to the Creative Commons licence, and indicate if changes were made. The images or other third party material in this article are included in the article's Creative Commons licence, unless indicated otherwise in a credit line to the material. If material is not included in the article's Creative Commons licence and your intended use is not permitted by statutory regulation or exceeds the permitted use, you will need to obtain permission directly from the copyright holder. To view a copy of this licence, visit http://creativecommons.org/licenses/by/4.0/ The Creative Commons Public Domain Dedication waiver (http://creativecommons.org/publicdomain/zero/1.0/) applies to the data made available in this article, unless otherwise stated in a credit line to the data. 


\section{Background}

Periodontitis is an inflammatory disease induced by the dental biofilm and affecting the tooth supporting tissues, bone and connective tissues, which may result in tooth loss. Several bacterial species have been associated with periodontitis and been designated major periodontopathogens [1]. Aggregatibacter actinomycetemcomitans has been associated with periodontitis in both young and older individuals $[2,3]$. A. actinomycetemcomitans produces a leukotoxin (Ltx), an exotoxin targeting cells of the immune system in the periodontal tissues [4]. Leukotoxin induces a pro-inflammatory response by activation of macrophages/ monocytes and secretions of IL-1 $\beta$, and also selectively kills human leukocytes [5]. The leukotoxic activity among $A$. actinomycetemcomitans strains and subtypes is variable, and a highly toxic clone has been identified [6]. This genotype, termed the JP2 clone, primarily found in North and West African populations, has been closely associated with periodontitis in young individuals [6,7]. Previous studies on various subtypes of $A$. actinomycetemcomitans strains isolated from an adult Thai population with periodontitis, revealed a significant variation in IL-8 cytokine expression (but not IL-1 $\beta$, IL-6, and TNF- $\alpha$ ) in human gingival epithelial cells (HGECs) $[8,9]$. Non-serotypable strains (NS1 and NS2) and a JP2-like strain showed significantly lower IL-8 responses than the serotypable (serotype a-f) stains [9].

In the complex dental biofilm numerous interactions take place in order to regulate the microbial community by synergistic and antagonistic forces and metabolic networks [10]. Oral streptococci, which are abundant in most individuals, are considered to play a major role in regulating the dental biofilm ecology by producing interfering metabolic products and bacteriocins against a number of anaerobic gram-negative bacteria commonly associated with periodontitis including A. actinomycetemcomitans [11, 12]. Similarly, Lactobacillus species have shown inhibitory activities on the toxic effect of $A$. actinomycetemcomitans [13]. The purpose of the present study was to investigate the factors that are involved in the bacterial interaction between beneficial bacteria such as lactobacilli and a periodontopathogen such as $A$. actinomycetemcomitans. More specifically, the aim of this study was to examine the potential inhibiting or reducing effect of oral Lactobacillus strains on the cytotoxicity and secretion of the cytokines IL-1 $\beta$, IL-6, IL- 8 and TNF- $\alpha$ in peripheral blood mononuclear cells (PBMCs) by cell wall extracts (CWEs) and supernatants from a spectrum of $A$. actinomycetemcomitans subtypes.

\section{Results}

Cytotoxicity and IL-1 $\beta$ release from PBMCs by $A$. actinomycetemcomitans subtypes and Lactobacillus spp. The cytotoxicity and IL- $1 \beta$ release upon stimulation with supernatant and CWEs of individual strains of lactobacilli and A. actinomycetemcomitans strains are shown in Fig. 1. Treatment of PBMCs with supernatants from the three lactobacilli strains resulted in lower cytotoxicity, approximately $1.2-2.5$ folds (ranged 25.6 \pm 4.8 to $28.7 \pm 5.6 \%$ ), and IL-1 $\beta$ secretion, approximately $1.5-$ 3.3 folds (ranged $26.7 \pm 5.2$ to $36.9 \pm 1.3 \mathrm{pg} / \mathrm{mL}$ ), compared to when treated with $A$. actinomycetemcomitans strains NS1, NS2, JP2-like, and JP2 clone $(p<0.05$, Fig. 1a and b). Also, NS2, JP2-like, and JP2 clone showed significantly higher cytotoxicity and IL-1 $\beta$ secretion compared to serotype a, serotype c, NS1, ATCC33384 (serotype c) and ATCC29523(serotype a) $(p<0.05$, Fig. $1 \mathrm{a}$ and $\mathrm{b})$. The significance for the higher release of IL$1 \beta$ by the JP2 clone (OMG3952) in comparison with ATCC 33384 and ATCC 29523 was $p<0.041$ and $p<$ 0.026 respectively.

The percentage of cytotoxicity and IL-1 $\beta$ secretion induced by CWEs from $A$. actinomycetemcomitans strains was higher than lactobacilli strains, approximately 1.43.4 folds (ranged $50.4 \pm 7.4$ to $70.6 \pm 2.1 \%$ ) and $1.4-3.1$ folds (ranged $31.7 \pm 13.6$ to $194.3 \pm 45.4 \mathrm{pg} / \mathrm{mL}$ ), respectively. Also, NS1, NS2, JP2-like, and JP2 clone showed significantly higher cytotoxicity and IL-1 $\beta$ secretion than serotype a, serotype c, ATCC33384 (serotype c) and ATCC29523 (serotype a) ( $p<0.05$, Fig. 1c and d).

The results of PBMCs treated with CWEs of tested strains were in line with the outcome of supernatant treatment by that $A$. actinomycetemcomitans strains and showed significantly greater cytotoxicity and IL-1 $\beta$ induction compared to lactobacilli strains (Fig. 1c and d).

The effect of Lactobacillus spp. on the cytotoxicity and IL$1 \beta$ release from PBMCs by $A$. actinomycetemcomitans

The supernatant of Lactobacillus strains showed a varied reducing capacity on $A$. actinomycetemcomitans induced cytotoxicity and IL-1 $\beta$ release on PMBCs from six blood donors. L. paracasei SD1 reduced both cytotoxicity and IL-1 $\beta$ release from PBMCs by $A$. actinomycetemcomitans strains (mean value of 8 strains) significantly approximately $0.5-1.3$ folds (from $58.9 \pm 4.7$ to $51.5 \pm 3.7 \%$ and $0.7-1.2$ folds $(42.9 \pm 4.6$ to $29.1 \pm 3.0 \mathrm{pg} / \mathrm{mL} ; p<0.05$; Fig. 2a and b). The CWEs of $L$. paracasei SD1 showed in line with supernatant a $0.8-1.7$ fold decreased cytotoxicity (from $57.6 \pm 5.3$ to $53.9 \pm 2.7 \%, p<0.05$; Fig. $2 \mathrm{a}$ ) and $0.5-1.2$ fold reduction of IL-1 $\beta$ secretion (from $174.0 \pm$ 32.8 to $143.1 \pm 27.3 \mathrm{pg} / \mathrm{mL}, p<0.001$; Fig. 2b) of $A$. actinomycetemcomitans CWEs. Also, L. rhamnosus SD11 reduced significantly IL- $1 \beta$ secretion by $A$. actinomycetemcomitans CWEs $0.7-1.2$ fold (from $174.0 \pm 32.8$ to $151.8 \pm 28.9 \mathrm{pg} / \mathrm{mL}, p<0.001$; Fig. $2 \mathrm{~b})$. The cytotoxicity and IL-1 $\beta$ secretion did not reach statistical significance for $A$. actinomycetemcomitans CWEs combined with CWEs of LGG (0.6-0.9 fold reduction of cytotoxicity and $0.7-0.9$ fold reduction of IL- $1 \beta$ secretion from 

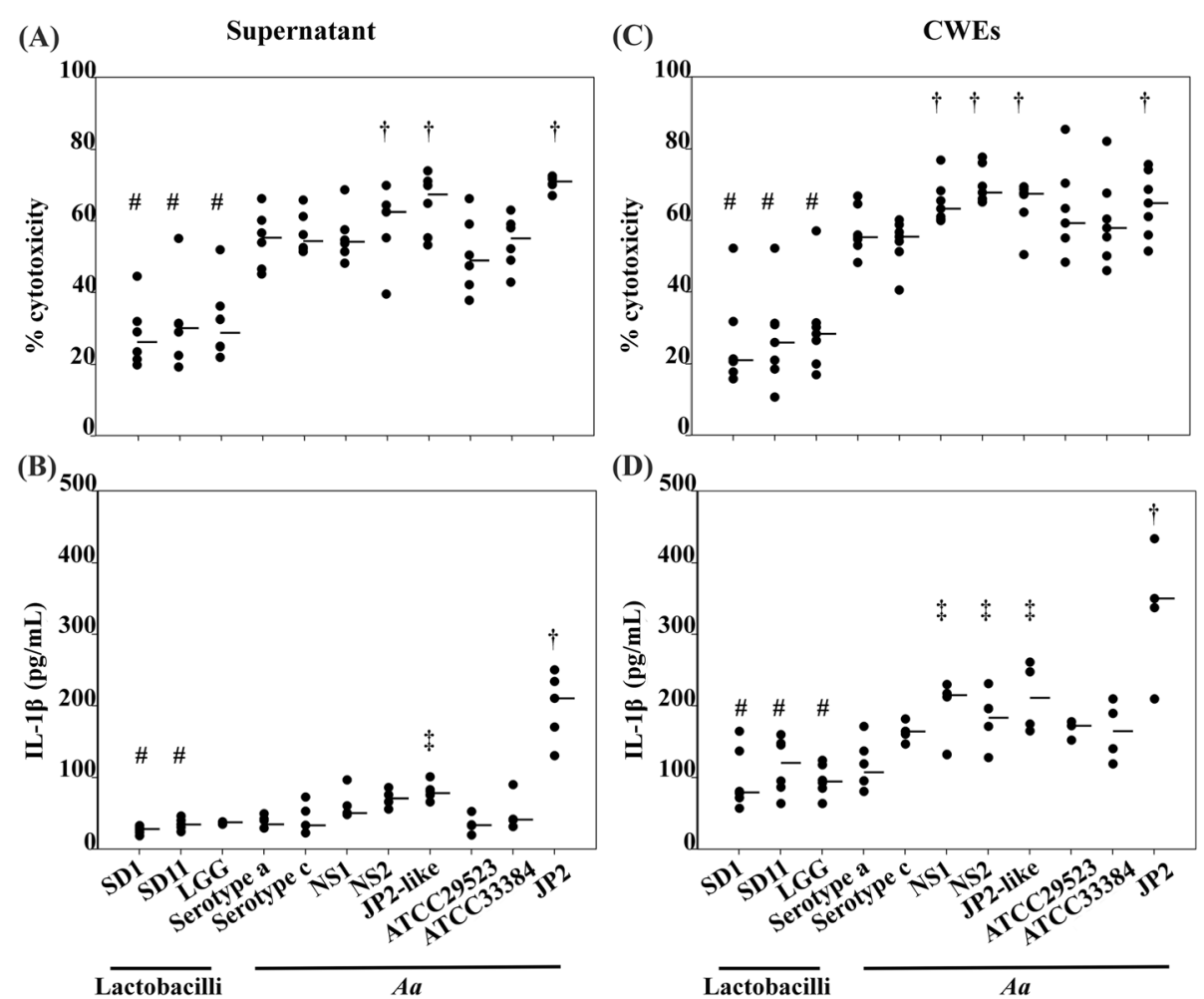

Fig. 1 The cytotoxicity ( $\mathbf{a}$ and $\mathbf{c}$ ) and IL-1 $\beta$ secretion ( $\mathbf{b}$ and $\mathbf{d}$ ) of the PBMCs exposed to the supernatant and CWEs of lactobacilli and $A$. actinomycetemcomtians, respectively. "Statistically significant lower cytotoxicity $(p<0.001)$ or IL-1 $\beta$ secretion $(p<0.042)$ of lactobacilli compared to A. actinomycetemcomtians, ${ }^{\dagger}$ Statistically significant higher cytotoxicity of $A$. actinomycetemcomtians strain NS1, NS2, JP2-like, and OMG3952 ( $p<$ $0.001)$ and IL-1 $\beta$ secretion of $A$. actinomycetemcomtians strain OMG3952 compared to other strains $(p<0.001)$, ₹ Statistically significant higher IL$1 \beta$ secretion of $A$. actinomycetemcomtians strain NS1, NS2 and JP2-like, except OMG3952, compared to other strains $(p<0.045)$. The statistical method was Mann Whitney $U$ test and the median of the group is shown as a horizontal line

$174.0 \pm 32.8$ to $164.5 \pm 42.5 \mathrm{pg} / \mathrm{mL} ; p>0.05$; Fig. $2 \mathrm{a}$ and b).

\section{Effect of Lactobacillus paracasei SD1 on the cytotoxicity and IL-1 $\beta$ release by CWEs from $A$. actinomycetemcomitans subtypes}

The cytotoxicity of individual $A$. actinomycetemcomitans strains after combination with $L$. paracasei SD1 was significantly lower compared with $A$. actinomycetemcomitans alone $(p<0.01 ;$ Fig. 4$)$. The reducing effect of $L$. paracasei SD1 was 1.0-1.6 folds. NS1, NS2 and JP2-like strains revealed significant reduction compared to each of them alone showing 1.0-1.4 folds of reduction (Fig. $4)$. Individual variations on the response of the PBMCs were however, noticed.

The ability of $L$. paracasei SD1 to reduce cell responses induced by separate A. actinomycetemcomitans strains is shown in Fig. 3 and Fig. 4. The CWEs of $L$. paracasei SD1 proved to have a significant and consistent negative impact on IL-1 $\beta$ secretion in all cell cultures treated with the different A. actinomycetemcomitans strains $(p<0.01)$. The adding of L. paracasei SD1 to the cell cultures reduced the effect of each $A$. actinomycetemcomitans strain approximately 1.0-11.7 folds compared to when the cells were cultured with $A$. actinomycetemcomitans alone (Fig. 3). NS1, NS2, and JP2-like (ranged 128.1 to $261.0 \mathrm{pg} / \mathrm{mL}$ ) showed a significant reduction approximately $1.2-10.2$ folds compared with the addition of $L$. paracasei SD1 (ranged 25.6 to $174.3 \mathrm{pg} / \mathrm{mL}$ ) in all subjects. Also, here, large individual variations were seen between the PBMCs.

Effect of $A$. actinomycetemcomitans subtypes alone and combination with $L$. paracasei SD1 on IL-6, IL-8 and TNF-a release from PBMCs

TNF- $\alpha$ (ranged 1256.6 to $51,103.8 \mathrm{pg} / \mathrm{ml}$ ) showed the high levels when exposed to $A$. actinomycetemcomitans strains (Fig. 5) while IL-6 (ranged 10.34 to $631.9 \mathrm{pg} / \mathrm{ml}$ ) and IL-8 (ranged 1009.9 to $15,480.6 \mathrm{pg} / \mathrm{mL}$ ) were considerably lower than TNF- $\alpha$ (Fig. 6 and Fig. 7). After combination, $L$. paracasei SD1 reduced the secretion of IL-6, IL-8, and TNF- $\alpha$ significantly in all cell cultures also treated with the different $A$. actinomycetemcomitans strains $(p<0.05$; Figs. $5,6,7)$. The reduced secretion of IL-6, IL-8, and TNF- $\alpha$ was $1.1-20.7,1.1-3.4$, and 1.1- 


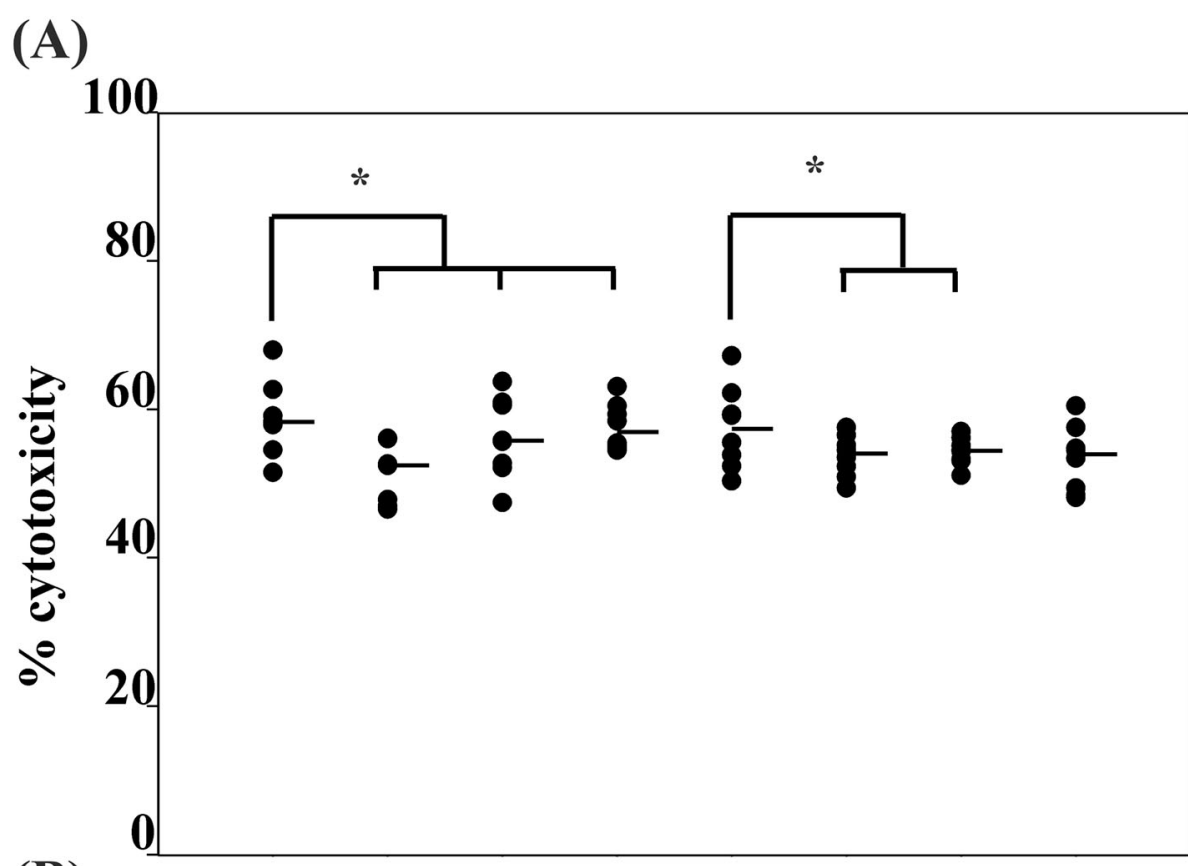

(B)

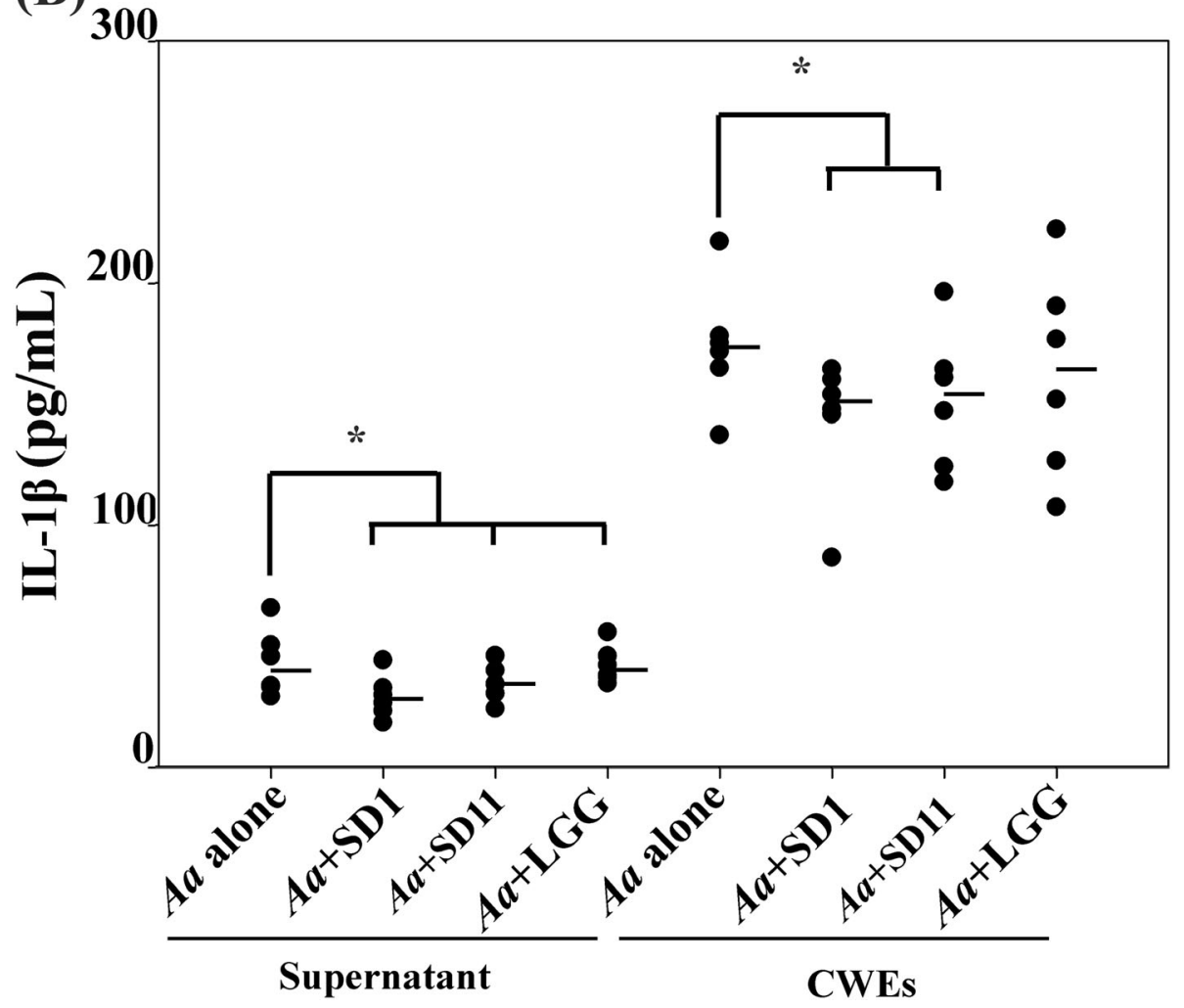

Fig. 2 The cytotoxicity (a) and IL-1 $\beta$ concentration (b) secreted from PBMCs (from six blood donors) exposed to the supernatant (1:1 concentration) or CWEs $(100 \mu \mathrm{g} / \mathrm{mL})$, where each spot represents one blood donor and a mean value of eight $A$. actinomycetemcomitans strains alone or in combination with three strains of Lactobacillus spp. (SD1, SD11 or LGG). * Statistically significant lower cytotoxicity $(p<0.001)$ or IL-1 $\beta$ concentration $(p<0.048)$ for the combinations compared to A. actinomycetemcomitans alone using Mann Whitney $U$ test. The median of the group is shown as a horizontal line 


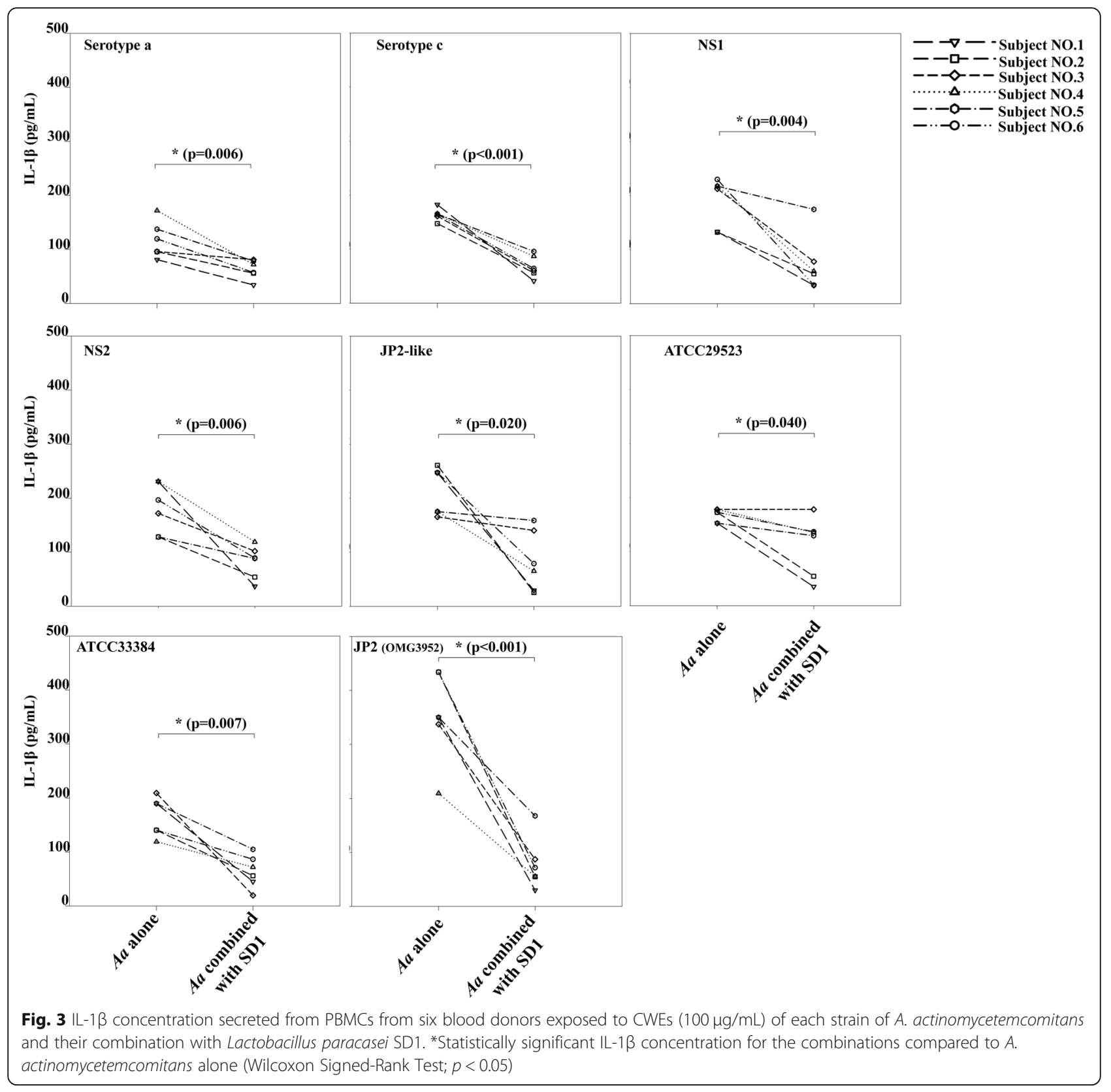

11.5 folds, respectively. Strong individual variations were also seen here.

\section{Discussion}

This study examined the effect of oral lactobacilli on the cytotoxicity and pro-inflammatory cytokine responses of PBMCs induced by eight different subtypes of $A$. actinomycetemcomitans. The main finding was that statistically significant reductions of the cytotoxicity and the proinflammatory cytokines, IL- $1 \beta$, IL-6, IL- 8 , and TNF- $\alpha$ was demonstrated for oral Lactobacillus strains, and $L$. paracasei SD1 in particular, when combined with the $A$. actinomycetemcomitans subtypes although a significant variation between the blood donors was noticed. The study illustrates the high complexity in the interaction between oral bacteria and the host that exists even in vitro between bacterial species, species subtypes and components and between host (blood donor) cells such as PBMCs.

All A. actinomycetemcomitans and Lactobacillus strains showed a toxic effect and induced the release of cytokines from PBMCs in various degrees, although A.actinomycetemcomitans showed a generally and statistically significant higher toxic effect than the Lactobacillus strains. This finding was for both CWEs and supernatant indicating that various cell wall components 


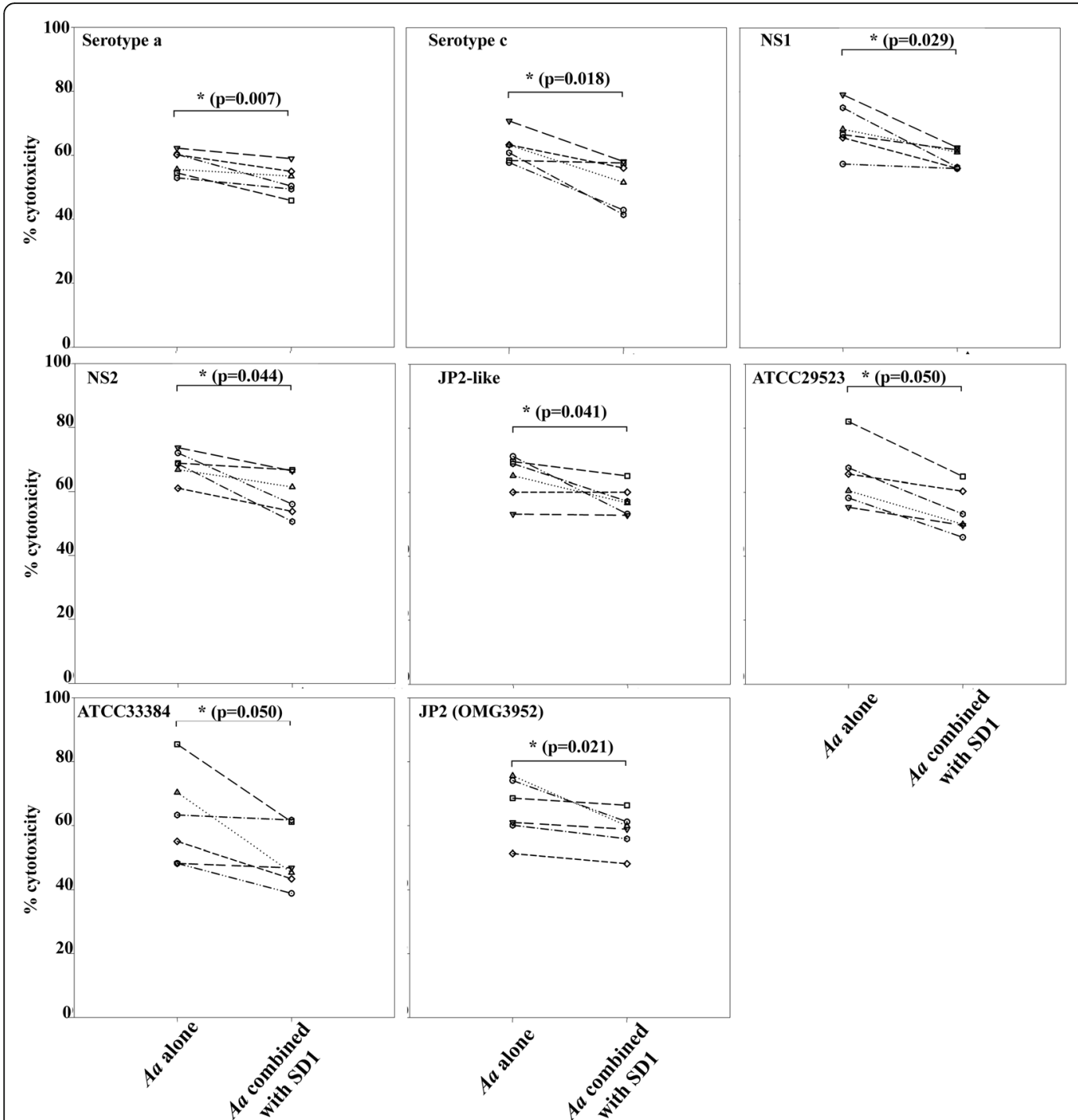

Fig. 4 Cytotoxicity of PBMCs from six blood donors exposed to CWEs $(100 \mu \mathrm{g} / \mathrm{mL})$ of each strain of $A$. actinomycetemcomitans and their combination with Lactobacillus paracasei SD1. *Statistically significant cytotoxicity for the combinations compared to A. actinomycetemcomitans alone (Wilcoxon Signed-Rank Test; $p<0.05$ )

as well as extracellular products from all bacteria, gramnegative as well as gram-positive, interfere with the host cells, in this case PBMCs. This means that the cytotoxicity as was measured in this study is of low specificity and basically not related to any particular component of the bacteria. This should be kept in mind for the complex in vivo situation with a dental biofilm with hundreds of different species in highly variable amounts. Thus, the effect of specific bacterial species, such as $A$. actinomycetemcomitans or lactobacilli, is generally unpredictable.

Not surprisingly the highly, according to the literature [6], toxic genotype JP2 of A actinomycetemcomitans showed the highest toxic effect and significantly higher than the other A. actinomycetemcomitans subtypes.
Similarly, the effect on IL-1 $\beta$ release was more pronounced for the JP2 clone, indicating that the effect on host cells may be specifically associated with leukotoxin production and with a potential co-stimulation of PBMCs by lipopolysaccharides (LPS). A. actinomycetemcomitans is a bacterial species associated with periodontitis due to its leukotoxin production, internalization into fibroblast cells, and immunomodulation $[8,9]$. In a previous study it was shown that $A$. actinomycetemcomitans suppressed IL-8 mRNA expression and its function [9] as well as, this study revealed the potency of $A$. actinomycetemcomitans on stimulating PBMCs to secret IL1ß. Lipopolysaccharide (LPS) [9] and leukotoxin [14] of A. actinomycetemcomitans are immunodominant components that stimulate immune cell produced pro- 


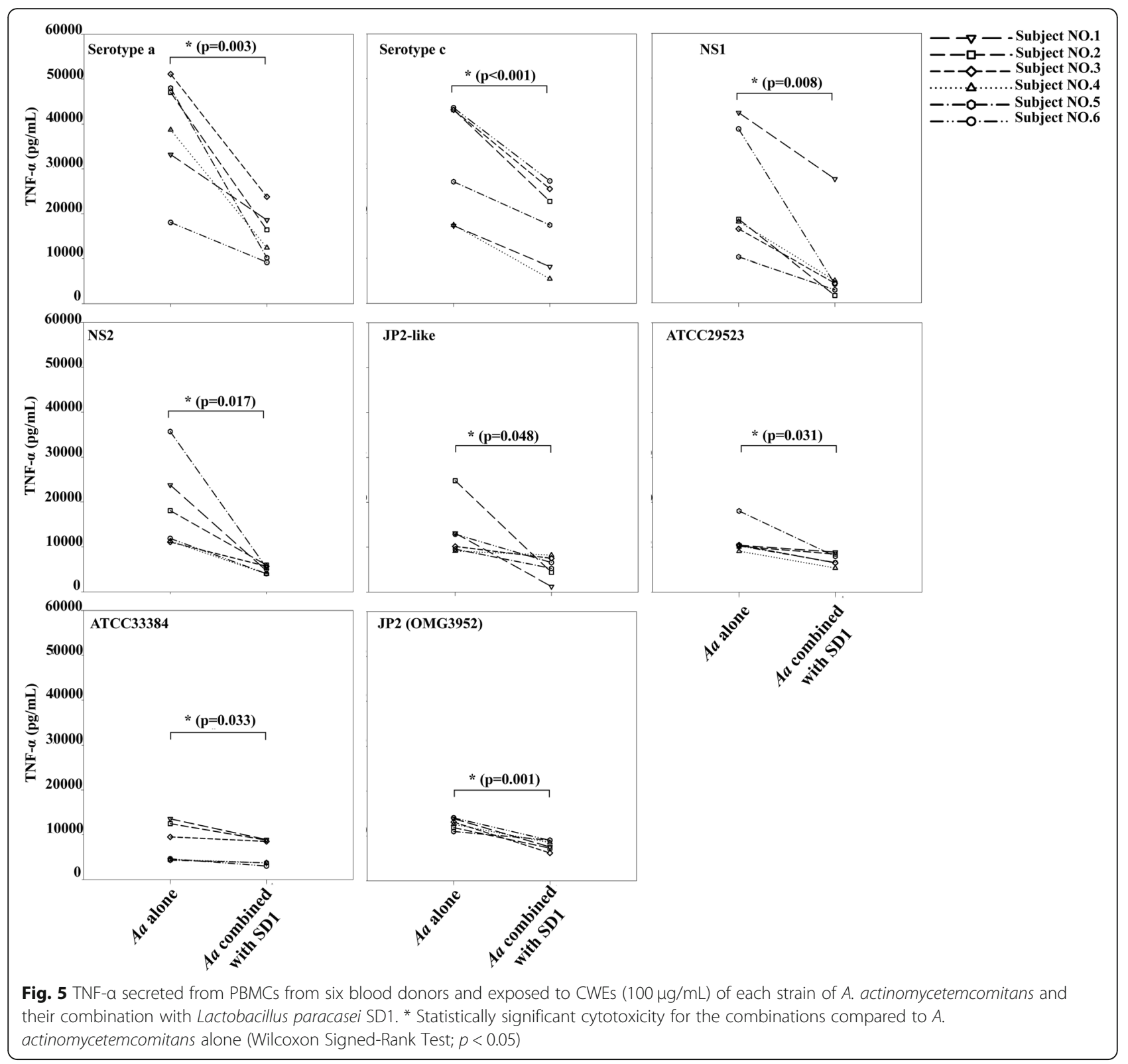

inflammatory cytokines and chemokine, which is in accordance with the results of this study. A. actinomycetemcomitans CWEs and supernatant showed significantly higher cytotoxicity and IL- $1 \beta$ secretion by PBMCs compared to lactobacilli. The response to the highly toxic JP2 genotype (OMGS 3952) indicates that the leukotoxin plays a major role in the IL- $1 \beta$ release from PBMCs.

A. actinomycetemcomitans components other than the toxin appeared to be stronger regulators of proinflammatory cytokines such as IL-6, IL-8, TNF- $\alpha$ in the various experiments. The release of the cytokines IL- 6 and IL-8 was low to moderate (Fig. 6 and Fig. 7) while the release of TNF- $\alpha$ was remarkably high for all $A$. actinomycetemcomitans subgroups (Fig. 5). The JP2 subtype (strain OMG3952) showed here the lowest response except for one of the blood donors (Fig. 5, OMGS 3952), indicating that other bacterial components than leukotoxin may be responsible for the TNF- $\alpha$ release.

Both supernatants and CWEs of oral lactobacilli reduced the cytotoxicity and pro-inflammatory cytokines of the PBMCs when combined with an adjusted amount of supernatant and CWEs of A. acinomycetemcomitans strains. In particular, the supernatant and CWEs of $L$. paracasei SD1 had a significant and consistent reduction on IL- $1 \beta$, IL- 6 , IL- 8 , and TNF- $\alpha$ release on all subtypes of $A$. acinomycetemcomitans. The component responsible for the reducing effect in lactobacilli is not known. 


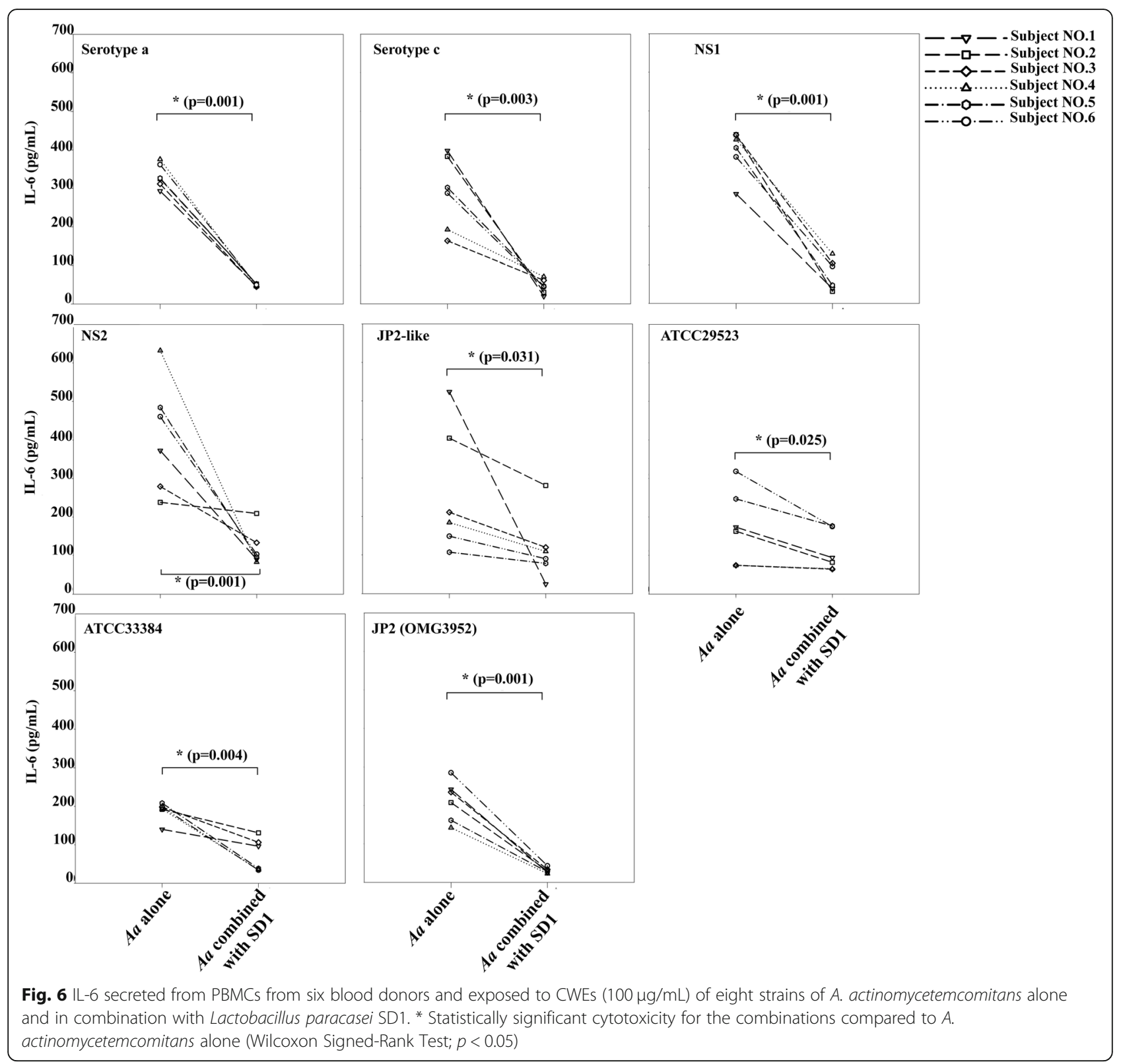

Lactobacillus strains have been recognized as beneficial bacteria along the gastrointestinal tract and suggested and used as a probiotic due to its both general inhibiting effect on other microorganisms e.g. by its acid production $[10,11]$ and specific effect on bacterial growth [15] and reducing effect on toxicity by bacteriocins [13]. The potential interactions in the regulation of the dental biofilm ecology are numerous and involve many different microbial species and the importance of specific interaction between lactobacilli and A. actinomycetemcomitans, as was shown in this in vitro study, can only at this stage be speculated. The reducing effect of the oral Lactobacillus strains on the toxicity of A. actinomycetemcomitans could be considered limited from this in vitro study in view of the rare colonization of lactobacilli in the periodontal pocket, however it is possible that certain strains may interact with the colonization and growth of $A$. actinomycetemcomitans in the early stages of periodontal disease.

The response was highly variable between the blood donors for both IL- $1 \beta$ and TNF- $\alpha$ and even nonresponders were noticed as illustrated by subject 6 (Fig. 3 ) in whom IL-1 $\beta$ was not detected for any of the 8 subtypes of $A$. actinomyctemcomitans. This variation in host susceptibility for a putative periodontal pathogen such as A. actinomycetemcomitans and cell components and extracellular products from them illustrates the complexity of the interaction between the dental biofilm and 

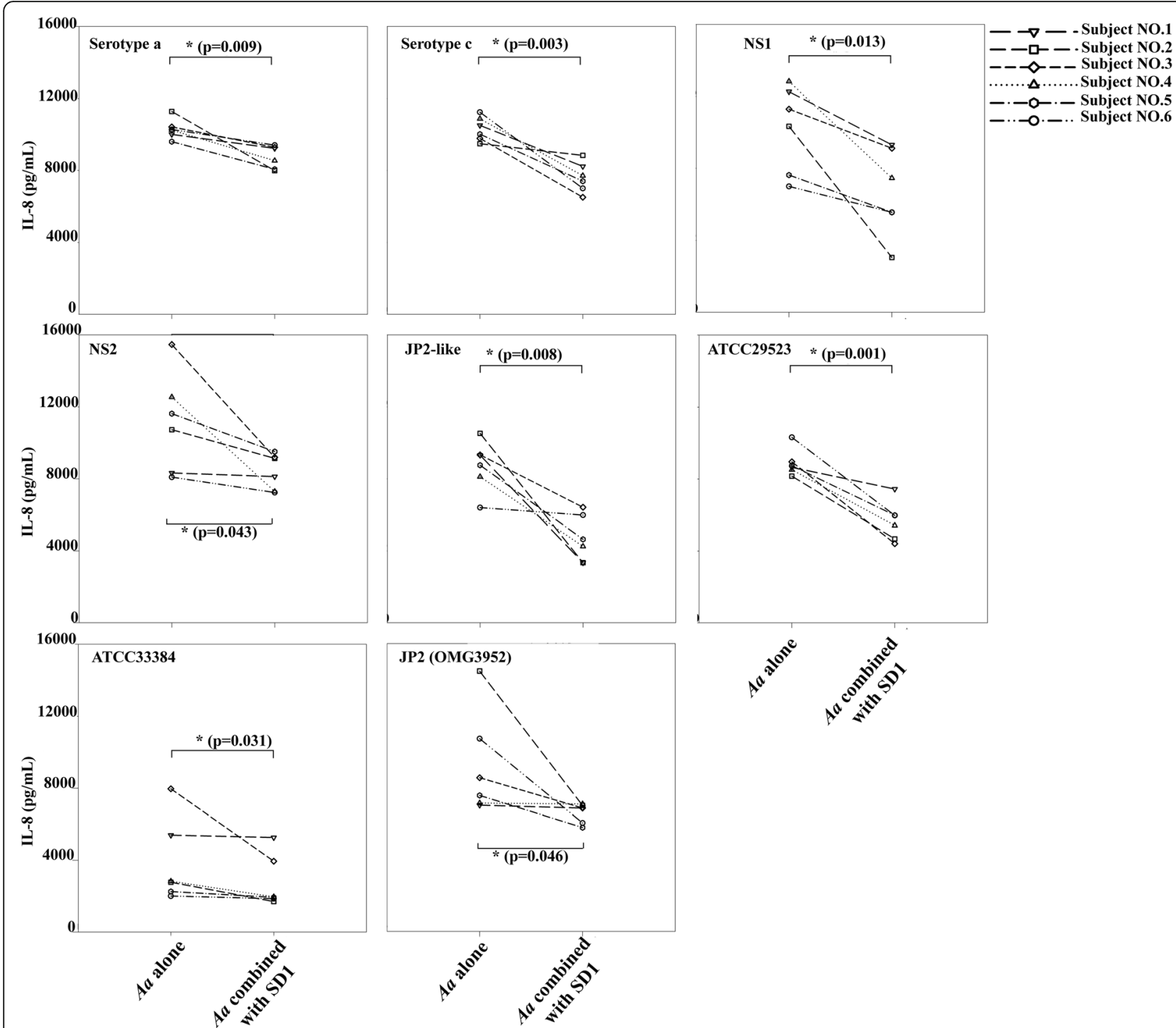

Fig. $7 \mathrm{IL}-8$ secreted from PBMCs from six blood donors and exposed to CWEs (100 $\mu \mathrm{g} / \mathrm{mL})$ of eight strains of $A$. actinomycetemcomitans alone and in combination with Lactobacillus paracasei SD1. * Statistically significant cytotoxicity for the combinations compared to $A$.

actinomycetemcomitans alone (Wilcoxon Signed-Rank Test; $p<0.05$ )

host tissues in vivo and the variation in the periodontal disease susceptibility. It could be argued that the variation seen between the blood donors may be due to methodological errors in collecting, storage and preparing the buffy coats for the experiments. All buffy coats were ordered and prepared from blood samples the day before the experiment. At the day of the experiment, the cells from various buffy coats were treated in parallel and in same and standardized way. We therefore argue that methodological errors have only a minor impact in this study. Instead, this study show that substantial and inter-individual variation exists in cytokine release from the monocytes. Thus, there is a benefit of using buffy coats in studies to include this natural variation among different subjects, which is not possible when using cell lines.

\section{Conclusions}

This study found a limited but statistically significant reducing effect of three oral Lactobacillus strains on the toxicity of eight Aggregatibacter actinomycetemcomitans strains. This in vitro study further demonstrates the complexity of the interactions among bacteria and also between bacteria and the host cells. The large variability among the target cells (A. actinomycetemcomitans), among the effector strains (lactobacilli), and among host cells (PBMCs from blood donors), illustrate this complexity. The most inhibited strains were the most toxic 
target strains (the JP2 clone), and $L$. paracasei SD1 had a more inhibiting effect than the other oral Lactobacillus strains.

\section{Methods}

Study design

The following factors were tested:

-The toxicity and cytokine release for the individual strains of A. actinomyctemcomitans and Lactobacillus spp.

- The toxicity and cytokine release of supernatant and cell wall extract (CWEs) of each strain respectively.

- The toxicity and cytokine release of a strain of $A$. actinomycetemcomitans alone and in combination with Lactobacillus strains.

- The diversity of cytokine release between blood donors.

\section{Bacterial strains}

Five strains of $A$. actinomycetemcomitans were selected among clinical isolates from Thai adults with periodontitis and representing different subtypes (serotype a and c, a JP2 like serotype c, and 2 non-serotypable strains, NS1 and NS2, representing two different DGGE subtypes that have shown a high toxicity in a previous study [8]) were included as target strains for the study together with 3 reference strains representing serotype a (ATCC29523), serotype c (ATCC33384), and the JP2 clone serotype b (OMGS 3952). The latter stain was isolated from a young girl of Cap Verde Island with an advanced periodontitis [16]. Further, Lactobacillus paracasei SD1 and L. rhamnosus SD11 previously tested as oral probiotic strains $[17,18]$ and a reference strain $L$. rhamnosus ATCC53103 (LGG) were used as effector strains. The A. actinomycetemcomitans strains were cultured in brain heart infusion broth (BHI, Acumedia, Neogen, Lansing, Mich, USA) and the Lactobacillus strains in Lactobacillus selective medium (Acumedia, Neogen, Lansing, Mich, USA) and were incubated at $37^{\circ} \mathrm{C}$ for $48 \mathrm{~h}$ under anaerobic conditions.

\section{Bacterial supernatant preparation}

The concentrations of A. actinomycetemcomitans were adjusted by the optical dentistry at $\mathrm{OD}_{600}=0.15$ which corresponded to $10^{8} \mathrm{CFU} / \mathrm{mL}$ in BHI broth and were incubated at $37^{\circ} \mathrm{C}$ for $48 \mathrm{~h}$ under anaerobic conditions. Supernatants were collected after centrifugation at 2800 $\mathrm{g}$ for $10 \mathrm{~min}$ and then, the $\mathrm{pH}$ was adjusted to $\mathrm{pH} 7.0$. The supernatants were filtrated and stored at $-20^{\circ} \mathrm{C}$ until further analysis. The cell pellets were kept for cell wall extraction (see below).

The supernatant of Lactobacillus strains was prepared in the same way as for A. actinomycetemcomitans, although the concentration of Lactobacillus cells was adjusted at $\mathrm{OD}_{600}=0.20$ ) corresponding to cell counts of $10^{8} \mathrm{CFU} / \mathrm{mL}$.

\section{Bacterial cell wall preparation}

Cell pellets were used to extract cell wall components by differential centrifugation, as previously described [9]. Briefly, bacterial cells were resuspended in PBS pH 7.0 in the presence of a proteinase inhibitor cocktail (1 tablet yields a $1 \mathrm{mM}$ EDTA solution in $10 \mathrm{ml}$, Roche Molecular Biochemicals, Mannheim, Germany) and the cells were disrupted by sonication. Intact cells were removed by centrifugation at $2200 \mathrm{~g}$ for $10 \mathrm{~min}$ at $4{ }^{\circ} \mathrm{C}$, whereas the cell wall extract (CWEs) were collected from the supernatant by centrifugation at $30,000 \mathrm{~g}$ for $20 \mathrm{~min}$ at $4{ }^{\circ} \mathrm{C}$. The cell wall pellet was resuspended in $500 \mu \mathrm{L}$ of PBS with $\mathrm{pH} 7.0$, and the total protein concentration was determined by the Bradford assay [19].

\section{Human peripheral blood mononuclear cells (PBMCs) isolation}

Buffy coats were prepared from fresh blood collected from six healthy blood donors at hospital blood bank of the Sahlgrenska University Hospital in Gothenburg, Sweden and used for the experiments within $24 \mathrm{~h}$. The buffy coats were used after deidentification, and according to Swedish legislation section code $4 \mathbb{S} 3 p$ SFS 2003: 460 , no informed consent is needed. The PBMCs isolated from the buffy coat from each donor were used for each co-incubation with all tested bacteria to analyze cytotoxicity and cytokine secretions. The experiments were run in triplicates for each buffy coat.

Peripheral blood mononuclear cells (PBMCs) were isolated from buffy coats by centrifugation over FicollPaque $^{\mathrm{Tm}}$ Plus density gradient (GE healthcare BioSciences AB, Uppsala, Sweden). In brief, each buffy coat was diluted 1:1 with PBS pH 7.0 and layered on $3 \mathrm{ml}$ of Ficoll-Paque and centrifuged at $400 \mathrm{~g}$ for $30 \mathrm{~min}$ at room temperature. The PBMCs were collected at the interphase and washed twice in PBS and finally resuspended in Dulbecco's Modified Eagle Medium plus GlutaMAX ${ }^{\mathrm{mu}}$ (Gibco, Life Technologies, Paisley, UK) supplemented with 5\% human serum (Sigma-Aldrich, St Louis, MO, USA) and $1 \%$ penicillin-streptomycin (Sigma-Aldrich), then the cells were counted using a hematocytometer.

\section{Bacterial exposure}

The PBMCs were added to 96-well plates at $2 \times 10^{6}$ cells/well and cultured in the presence of the various concentrations $(50,100,200,400,800,1000 \mu \mathrm{g} / \mathrm{mL})$ of lactobacilli CWEs and tested for cytotoxicity and cytokine secretion in order to find a suitable concentration for the experiments. A concentration of $100 \mu \mathrm{g} / \mathrm{mL}$ of lactobacilli CWEs was used while the concentration of A. actinomycetemcomitans CWEs was obtained from our 
previous study [9]. For the supernatant of both strains, an undiluted supernatant was used throughout this study.

PBMCs were added to 96-well plates at $2 \times 10^{6}$ cells/ well and cultured in the presence of bacterial supernatants or $100 \mu \mathrm{g} / \mathrm{mL}$ CWEs of either A. actinomycetemcomitans or Lactobacillus strains alone or combinations for $2 \mathrm{~h}$ at $37^{\circ} \mathrm{C}$ in $5 \% \mathrm{CO}_{2}$ incubator. PBMCs cultured without bacterial components were used as controls. The cell-free culture supernatants were collected for IL$1 \beta$, IL- 6 , IL-8, and TNF- $\alpha$ determination and the PBMC cells were collected for the cytotoxicity test.

\section{Cytotoxicity and IL-1 $\beta$ determination}

The cytotoxicity of A. actinomycetemcomitans or Lactobacillus strains, single or combinations, was determined using the modified trypan blue exclusion method [20]. The percentage of cytotoxicity was calculated by 100 - (surviving cells of the test/surviving cells of the control $\times 100$ ).

IL-1 $\beta$ analysis of cell-free culture medium was performed using the DuoSet ELISA Development Kit (R\&D Systems, Abingdon, UK) according to the manufacturer's instructions. The plates were coated with capture antibody overnight. The cell culture supernatants were incubated with a cytokine-specific biotinylated detection antibody and marked with streptavidin-conjugated horseradish-peroxidase. After the addition of the substrate, the absorbance was measured using an ELISA microplate reader (Synergy 2, BioTek Instruments, Inc., Winooski, VT, USA) at $405 \mathrm{~nm}$ and compared to a standard curve in order to calculate the concentration presented as $\mathrm{pg} / \mathrm{mL}$.

\section{IL-6, IL-8, and TNF- $\alpha$ determination}

The cytokines, IL- 6 , IL- 8 , and TNF- $\alpha$, were measured using a custom-made multiplex assay (Bio-Plex Express Assay, Bio-Rad Laboratories, Hemel Hempstead, UK) based on Luminex xMAP technology according to the manufacturer's instructions. Briefly, the standard was reconstituted and diluted in a fourfold dilution series. Antibody coupled capture beads were prepared and plated. After washing using a Bio-Plex Pro $^{\text {tw }}$ wash station (Biorad), diluted samples and standards were added to the beads in the wells. The plate was incubated on a shaker and after incubation and wash, detection antibodies were added to each well and after the streptavidin-phycoerythin solution (R\&D Systems, Abingdon, UK) was added to the wells. In the last incubation step, beads were resuspended in assay buffer and the plate was read with a BioPlex 200 instrument equipped with BioManager analysis software (BioRad). The absolute concentrations of the samples were determined by comparing the bead colour and mean fluorescence intensity from each set of beads against an automatically optimized and manually verified standard curve. The cytokine concentration was presented as $\mathrm{pg} / \mathrm{mL}$.

\section{Statistical analysis}

The results of bacterial CWEs and supernatant on PBMCs stimulation were compared using Mann-Whitney U Test while the results of SD1 combined with A. actinomycetemcomitans on PBMCs stimulation were analyzed with Wilcoxon Signed-Rank Test. A $p$-value $<0.05$ was considered as statistically significant.

\section{Abbreviations}

BHI: Brain heart infusion; CWE: Cell wall extract; DGGE: Denaturated gradient gel electrophoresis; HGEC: Human gingival epithelial cells; IL: Interleukin; LPS: Lipopolysaccharide; NS: Non-serotypable; PBMCs: Peripheral blood mononuclear cells; TNF-a: Tumor necrotic factor-alfa

\section{Acknowledgements}

Special thanks for technical assistances in the Department of Oral Microbiology and Immunology, University of Gothenburg, Sweden.

\section{Authors' contributions}

NP, RT and GD designed the study. NP performed the experiments and drafted the manuscript. NP, AB, AKO, RT and GD discussed the study design and data analysis and revised the manuscript. All authors read and approved the final manuscript.

\section{Funding}

This study was supported by Research Grant from Public Dental Service (TUA) and Swedish Dental Society covering laboratory expenses. The Department of Oral Microbiology and Immunology at the Institution of Odontology, Sahlgrenska Academy, University of Gothenburg, Sweden funded the design of the study, collection analysis, interpretation of data and in writing the manuscript. A special grant was used from the international exchange program for travel expenses and accommodation for Dr. Pahumunto as a guest lecturer at Sahlgrenska Academy, University of Gothenburg, Gothenburg, Sweden. Open Access funding provided by University of Gothenburg.

Availability of data and materials

All data generated or analyzed during this study are included in this published article.

\section{Ethics approval and consent to participate}

Ethics approval not applicable. Buffy coats were obtained from the hospital blood bank at Sahlgrenska University Hospital, Gothenburg, Sweden after deidentification, and according to the Swedish legislation section code $4 \S$ 3p SFS 2003:460, no informed consent is needed. Permission to use bloodcomponents for other purposes than transfusion was approved (171017) with the reference number (KITM) K 22/17.

\section{Consent for publication \\ Not applicable.}

\section{Competing interests}

The authors declare that they have no competing interests.

\section{Author details}

${ }^{1}$ Common Oral Diseases and Epidemiology Research Center and Department of Stomatology, Faculty of Dentistry, Prince of Songkla University, Hat Yai, Thailand. ${ }^{2}$ Department of Oral Microbiology and Immunology, Institute of Odontology, Sahlgrenska Academy, University of Gothenburg, Gothenburg, Sweden. 
Received: 27 February 2020 Accepted: 27 August 2020

Published online: 11 September 2020

\section{References}

1. Socransky SS, Haffajee AD, Cugini MA, Smith C, Kent RL Jr. Microbial complexes in subgingival plaque. J Clin Periodontol. 1998;25:134-44.

2. Minguez M, Pousa X, Herrera D, Blasi A, Sanchez MC, Leon R, Sanz M. Characterization and serotype distribution of Aggregatibacter actinomycetemcomitans isolated from a population of periodontitis patients in Spain. Arch Oral Biol. 2014;59:1359-67.

3. Mombelli A, Casagni F, Madianos P. Can presence or absence of periodontal pathogens distinguish between subjects with chronic and aggressive periodontitis? A systematic review. J Clin Periodontol. 2002;29:10-21.

4. Johansson A. Aggregatibacter actinomycetemcomitans Leukotoxin: a powerful tool with capacity to cause imbalance in the host inflammatory response. Toxins. 2011;3:242-59.

5. Kelk P, Abd H, Claesson R, Sandström G, Sjöstedt A, Johansson A. Cellular and molecular response of human macrophages exposed to Aggregatibacter actinomycetemcomitans leukotoxin. Cell Death Dis. 2011;2:1-10.

6. Haubek D. The highly leukotoxic JP2 clone of Aggregatibacter actinomycetemcomitans: evolutionary aspects, epidemiology and etiological role in aggressive periodontitis. APMIS. 2010;118:1-53.

7. Åberg CH, Haubek D, Kwamin F, Johansson A, Claesson R. Leukotoxic activity of Aggregatibacter actinomycetemcomitans and periodontal attachment loss. PLoS One. 2014;9:1-11.

8. Pahumunto N, Ruangsri P, Wongsuwanlert M, Piwat S, Dahlen G, Teanpaisan R. Aggregatibacter actinomycetemcomitans serotypes and DGGE subtypes in Thai adult chronic periodontitis. Arch Oral Biol. 2015;60:1789-96.

9. Pahumunto N, Chotjumlong P, Makeudom A, Krisanaprakornkit S, Dahlen G, Teanpaisan R. Pro-inflammatory cytokine responses in human gingival epithelial cells after stimulation with cell wall extract of Aggregatibacter actinomycetemcomitans subtypes. Anaerobe. 2017;48:103-9.

10. Huang R, Li M, Gregory RL. Bacterial interactions in dental biofilm. Virulence. 2011;5:435-44.

11. Standar K, Kreikemeyer B, Redanz S, Munter WL, Laue M, Podbielski A. Setup of an in vitro test system for basic studies on biofilm behavior of mixed species cultures with dental and periodontal pathogens. PLoS One. 2010;5:1-14.

12. Duan D, Scoffield JA, Zhou X, Wu H. Fine-tuned production of hydrogen peroxide promotes biofilm formation of Streptococcus parasanguinis by a pathogenic cohabitant Aggregatibacter actinomycetemcomitans. Environ Microbiol. 2016;18:4023-36.

13. Nissen L, Sgorbati B, Biavati B, Belibasakis GN. Lactobacillus salivarius and L. gasseri down-regulate Aggregatibacter actinomycetemcomitans exotoxins expression. Ann Microbiol. 2014;64:611-7.

14. Kelk P, Claesson R, Chen C, Sjostedt A, Johansson A. IL-1 beta secretion induced by Aggregatibacter (Actinobacillus) actinomycetemcomitans is mainly caused by the leukotoxin. Int J Med Microbiol. 2008;298:529-41.

15. Jones SE, Versalovic J. Probiotic Lactobacillus reuteri biofilms produce antimicrobial and anti-inflammatory factors. BMC Microbiol. 2009;9:1-9.

16. Haubek D, Poulsen K, Westergaard J, Dahlen G, Kilian M. Highly toxic clone of Actinobacillus actinomycetemcomitans in geographically widespread cases of juvenile periodontitis in adolescents of African origin. J Clin Microbiol. 1996:34:1576-8

17. Teanpaisan R, Piwat S. Lactobacillus paracasei SD1, a novel probiotic, reduces mutans streptococci in human volunteers: a randomized placebocontrolled trial. Clin Oral Invest. 2014;18:857-62.

18. Teanpaisan R, Piwat P, Tianviwat S, Sophatha B, Kampoo T. Effect of longterm consumption of Lactobacillus paracasei SD1 on reducing mutans streptococci and caries risk: a randomized placebo-controlled trial. Dent J. 2015;3:43-54.

19. Bradford MM. A rapid and sensitive method for the quantitation of microgram quantities of protein utilizing the principle of protein-dye binding. Anal Biochem. 1979;72(1-2):248-54.

20. Pahumunto N, Ruangsri P, Wongsuwanlert M, Piwat S, Dahlen G, Teanpaisan R. Virulence of Aggregatibacter actinomycetemcomitans serotypes and DGGE subtypes isolated from chronic adult periodontitis in Thailand. Anareobe. 2015;36:60-4.

\section{Publisher's Note}

Springer Nature remains neutral with regard to jurisdictional claims in published maps and institutional affiliations.

Ready to submit your research? Choose BMC and benefit from:

- fast, convenient online submission

- thorough peer review by experienced researchers in your field

- rapid publication on acceptance

- support for research data, including large and complex data types

- gold Open Access which fosters wider collaboration and increased citations

- maximum visibility for your research: over $100 \mathrm{M}$ website views per year

At BMC, research is always in progress.

Learn more biomedcentral.com/submissions 